\title{
Traumatic subarachnoid haemorrhage: an alternative
} source

\author{
GC COAST, DJ GEE
}

From the Department of Forensic Medicine, St James's University Hospital, Leeds LS9 7TF

SUMMARY Traumatic subarachnoid haemorrhage is known on many occasions to result from a blow on the neck rupturing the vertebral artery within the cervical spine. On some occasions, however, no such damage to the artery in the neck can be found to account for the haemorrhage. Some cases are described in which the source of haemorrhage was rupture of the vertebral artery within the skull close to the basilar artery. The reason why rupture should occur at this site is discussed.

The possible association between fatal subarachnoid haemorrhage and injury has been appreciated for a long time. The exact source of bleeding, however, has not been so well established. At first, cases were thought to be due to rupture of an aneurysm of the circle of Willis; on occasions, such a ruptured aneurysm was found. Frequently, however, a source was not found, and it was then thought that a very small aneurysm must have been obliterated by the resultant haemorrhage. Proving that such a rupture was due to injury, and not due to a rise in blood pressure in the victim associated with excitement, was difficult.

In 1967 Simonsen' studied a large series of subarachnoid haemorrhages associated with violence and found that in such cases, especially where the source of bleeding was not found, there was a history of a fight, of intoxication by alcohol of the victim, and evidence of a blow to the head at the base of the skull.

The actual demonstration of the source of such haemorrhages, at an unexpected site, was first reported by Contostavlos, ${ }^{2}$ who as a result of careful dissection discovered dissecting aneurysms of the vertebral arteries associated with fracture of the transverse process of the first cervical vertebrae. His observations were soon confirmed by Cameron and Mant. ${ }^{3}$

As a result of these studies a protocol for the investigation of suspected traumatic subarachnoid haemorrhage was recommended. When a subarachnoid haemorrhage was the cause of death in someone who had died during a fight, and who had been

Accepted for publication 7 August 1984 drinking, the skin of the neck was scrutinised and a small fresh bruise just below one or other ear was often found. Arteriograms of the vertebral arteries were then carried out, either before or after the cervical spine and base of the skull were removed "en bloc." The arteriogram might show the site of leakage of the vertebral artery. Further examination proceeded either by removing all soft tissue from the spine, in order to show any fracture of the transverse processes of the cervical spine, or by exposing the vertebral artery throughout its length, so as to make possible a histological examination of the site of rupture of the artery. The type of injury giving rise to such a traumatic subarachnoid haemorrhage was usually a blow, either a kick or a punch, directed against the side of the neck, so as to cause the localised injury to the vertebral artery.

These discoveries appeared to provide a convincing, simple, and demonstrable explanation for the occurrence of traumatic subarachnoid haemorrhage. Therefore, during the past 12 years all such cases of suspected traumatic subarachnoid haemorrhage occurring in the practice of the Department of Forensic Medicine at Leeds have been examined by a regimen similar to that outlined above. An initial report of the results has been published ${ }^{4}$ : while some cases showed the typical features described by Contostavlos ${ }^{2}$ and Cameron and Mant ${ }^{3}$ in others no evidence of localised damage to a vertebral artery during its course within the cervical spine could be shown.

One such case (FM23537) when further examined was found to have a longitudinal tear in the wall of the vertebral artery, not in the neck but in that portion of the artery within the skull, lying in front of the pons, just above the point where the 
artery was severed to remove the brain from the skuil. Clearly, an injury in such a position could easily be obscured by being cut across when the brain was being removed, so that all the pathologist would then see would be a slightly ragged cut end of the artery, the importance of which could not be apparent. Therefore, in all subsequent cases of suspected traumatic subarachnoid haemorrhage the intracranial portion of the vertebral arteries has been reserved intact during the examination of the brain and spine. As a result, we have shown ruptures of the vertebral artery in this intracranial site, without any damage to the vertebral artery in the neck, in five of a total of 15 cases of traumatic subarachnoid haemorrhage seen during this period.

Attention has also been drawn to tears of the vessel at this site by Krauland ${ }^{5}$ and by Harland. ${ }^{6}$

The purpose of this paper is to draw attention to this site as an alternative to intracervical vertebral artery injury as an explanation for a traumatic subarachnoid haemorrhage, and to emphasise the ease with which such a rupture can be rendered undetectable by the standard procedure for removing the brain from the skull.

\section{Method}

In a case of sudden death during an altercation, if removal of the calvarium discloses a subarachnoid haemorrhage further dissection is postponed until after $x$ ray examination of the cervical spine and a vertebral arteriogram have been carried out with the brain in situ. Any bruises of the scalp are recorded and photographed. The sides of the neck below the ears are scrutinised for traces of bruising and then dissected so as to expose the top of the transverse process of the first cervical vertebra, and the adjacent muscles, in order to detect any bleeding into these tissues.

When the arteriogram has been completed the brain is removed by cutting through the upper end of the pons and through the lobes of the cerebellum, so as to the leave the pons, with the basilar artery, and below this the two vertebral arteries still attached to the base of the skull. The base of the skull is then sawn through in a rectangular outline, enclosing the foramen magnum, the inner halves of the middle fossae, and the sella turcica; the cervical spine at the level of $\mathrm{C} 5$ is divided transversely, and the upper part of the spine with the attached portion of the base of the skull is removed complete and fixed in formol-saline for some days before further examination.

After about one week the specimen is dissected so as to expose the vertebral arteries in the cervical spine, by removal of the anterior parts of the trans-

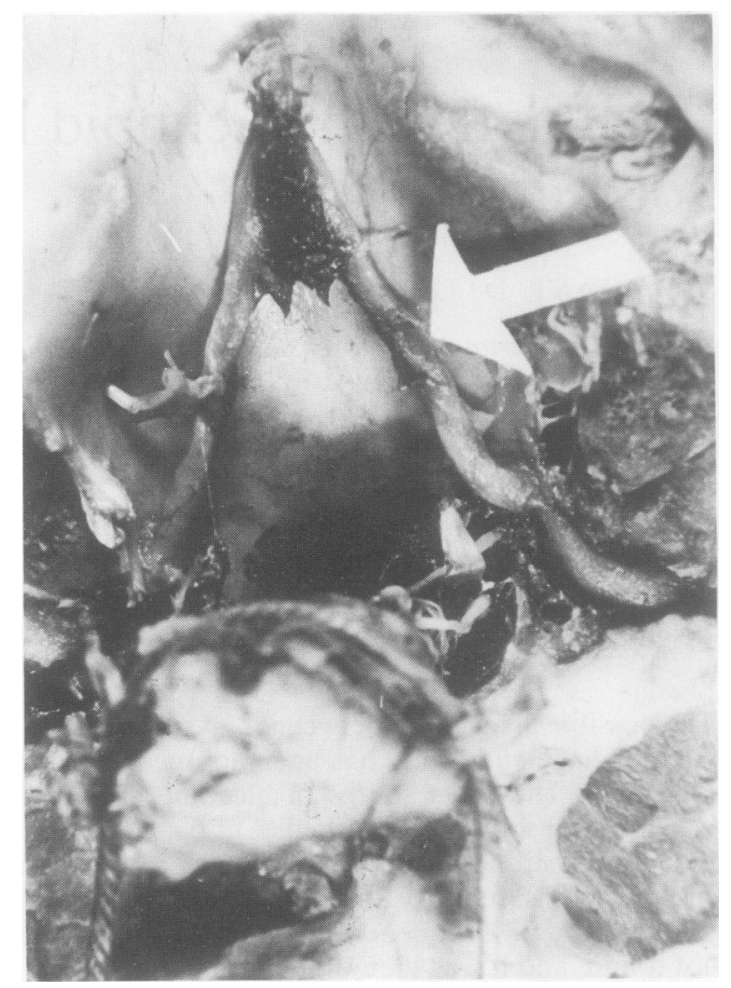

Fig. 1 Case 4 (FM30464X) showing position (arrow) of rupture of the right vertebral artery dissected in situ.

verse processes of the vertebrae. The course of each vertebral artery is traced through the floor of the skull, the intracranial portions of the arteries cleared of blood clot by blunt dissection with probe and forceps, and the vessels inspected.

\section{Results}

CASE 1 (FM21392A)

A 44 year old man, with a blood alcohol concentration of $148 \mathrm{mg} / 100 \mathrm{ml}$, collapsed and died during a fight in a pub in which a blow was aimed at the victim's head. Necropsy showed no bruising of the scalp and $x$ ray examination showed no fractures of the spine. Dissection revealed a $5 \mathrm{~mm}$ long tear in the left vertebral artery, within the skull, just above the point where the artery had been severed to remove the brain from skull. Microscopical examination of the site of rupture showed no evidence of arteritis. Dissection of the back of the neck showed a single bruise $2 \mathrm{~cm}$ in diameter low down in the neck.

\section{CASE 2 (FM23537)}

A 38 year old woman, with a blood alcohol concentration of $191 \mathrm{mg} / 100 \mathrm{ml}$, was involved in a domestic 
brawl with her husband. A single blow was said to have been struck, and there was bruising of the right forehead and right side of the nose. There was a large basal subarachnoid haemorrhage. $X$ ray examination showed no fracture of the bones or leakage from the vertebral arteries. Dissection of the neck revealed bleeding in the deep muscles at the right side of the neck extending downwards from the first cervical vertebra for $7 \cdot 5 \mathrm{~cm}$. A rupture was found in the wall of the right vertebral artery $3 \mathrm{~mm}$ above the point where it had been divided to remove the brain, in its intracranial course. The tear was $8 \mathrm{~mm}$ long and was directed longitudinally along the vessel wall.

\section{CASE 3 (FM24940)}

A 50 year old man had several bruises of the scalp and the left side of the face. An extensive subarachnoid haemorrhage was found over the base of the brain. A tear $6.5 \mathrm{~mm}$ long was found in the long axis of the left vertebral artery, in its intracranial portion, 2.5 $\mathrm{cm}$ above the point where it entered the skull. There was bleeding into the muscles in the left side of the neck from the level of the first cervical vertebrae downwards but no injury to the vertebral artery in its course within the neck.

\section{CASE 4 (FM30464X)}

A 17 year old boy, with a blood alcohol concentration of $152 \mathrm{mg} / 100 \mathrm{ml}$, was involved in a fight with several youths outside a club. There were a number of bruises of the scalp and an extensive basal subarachnoid haemorrhage. $X$ ray examination showed a leak of barium from the right vertebral artery apparently just above the transverse process of the first cervical vertebra. There was some bruising of the neck particularly in the region of the angle of the jaw on the right side. The cervical spine and the brain were removed from the skull so as to leave the vertebral arteries intact throughout the length of their course. Subsequent examination showed a longitudinal tear $6 \mathrm{~mm}$ long in the intracranial portion of the right vertebral artery within the skull $1 \mathrm{~cm}$ above the point where the artery passed through the dura. There was bleeding into the muscles around the first cervical vertebrae and in the canal surrounding the vertebral artery in the neck but no evidence of injury to the artery below the base of the skull. Microscopical examination of the artery at the site of rupture showed no evidence of inflammation (Figs. 1 and 2).

\section{CASE 5 (FM31521X)}

A 17 year old boy was involved in a fight in the street when he was punched and apparently kicked. He was taken to hospital unconscious and put on a

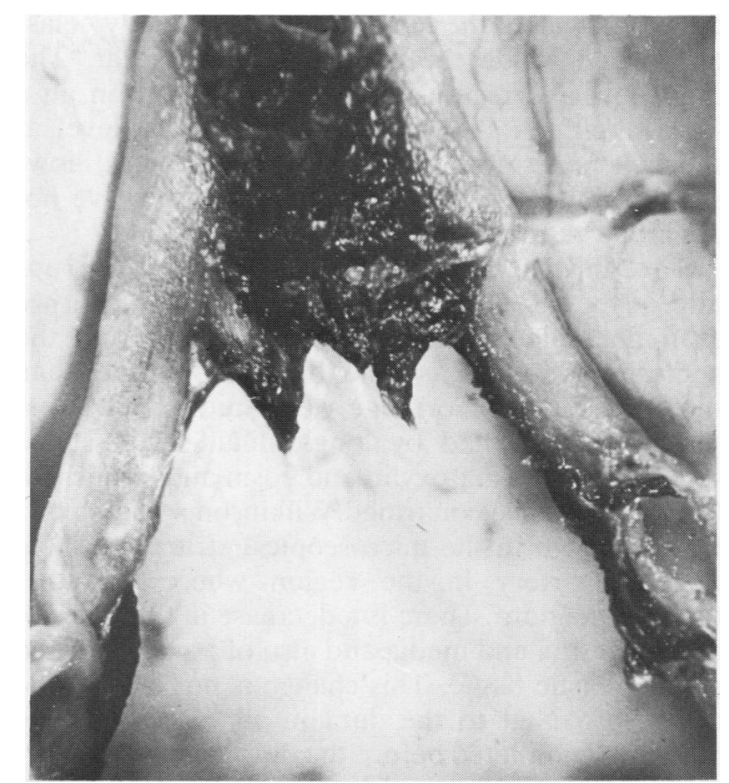

Fig. 2 Case 4 (FM30464X) Close up view of rupture in right vertebral artery.

life support machine but subsequently was found to be brain dead and the machine was switched off after two days. Postmortem examination revealed a single area of bruising of the scalp above the right eyebrow. There was bleeding into the right temporalis muscle and into the upper end of the right sternomastoid muscle. There was a large basal subarachnoid haemorrhage. $X$ ray examination showed no fracture of the cervical vertebrae but a leak of barium from the artery above the level of the floor of the skull. The brain and cervical spine were removed so as to retain the vertebral arteries throughout their length. A longitudinal rupture of the wall of the right vertebral artery was found in the intracranial portion $1 \mathrm{~cm}$ above the level where the artery pierces the dura; the laceration was $0.5 \mathrm{~cm}$ long. There was some bleeding along the course of the vertebral artery in its canal within the cervical spine, but no evidence of injury to the artery at that level. Microscopical examination at the site of rupture showed no evidence of inflammation. Further dissection of the left vertebral artery showed bleeding along its course in the upper part of the cervical spine but not associated with any injury to that artery at any point.

STRUCTURE OF THE VERTEBRAL ARTERY WALL Wilkinson' described a change in the structure of the wall of the vertebral artery between the portion of the vessel in the neck and the intracranial portion: 
the vessel wall in the neck was predominantly "elastic", while inside the skull it was muscular. The changeover occurred, according to Wilkinson, in a short length of the vessel roughly at the level at which it penetrated the dura. Other studies, however, such as that of Piffer and Zorzetto ${ }^{8}$ have not described these changes.

Since this might have relevance to the site of rupture of the vertebral arteries in the cases which we examined, nine vertebral arteries taken from the bodies of young adults dying from causes other than subarachnoid haemorrhage were studied. The vessels were examined by longitudinal sections and stained with haematoxylin and eosin and elastic van Geison. We have confirmed Wilkinson's finding of a major change in the microscopical structure of the vertebral artery in the region where it passes through the dura. There is a decrease in thickness of the adventitia and media and also of adventitial and medial elastic tissue. This change is not abrupt and begins proximal to the dura in all cases. In some cases it is completed before the dura, and, as Wilkinson found, it was completed in all cases within $15 \mathrm{~mm}$ distal to the dura.

\section{Discussion}

It seems likely that when a blow to the head causes a massive fatal subarachnoid haemorrhage the source is not always the same but may be due to damage to an artery in one of several different sites.

Thus it is well known that such an impact may cause an existing aneurysm of a cerebral artery to rupture. From the work of Contostavlos ${ }^{2}$ and Cameron and Mant $^{3}$ it is now well established that an injury to the side of the neck may cause rupture of a dissecting aneurysm of a vertebra in its course in the cervical spine. The method whereby the blood passes from the site of rupture in the neck through the dural attachment and base of the skull to reach the subarachnoid space is less certain. The lesion of the artery, however, seems to be due to a direct impact on the side of the neck.

In the case of rupture of the intracranial portion of the vessel, as described in this paper, it is obvious that the mechanism which causes rupture of the vessel cannot be direct trauma to the vessel itself. The possibility of traction due to sheering force causing rupture of vessels at the base of the brain was discussed by Tatsuno and Lindenberg.'

It is noteworthy that in the five cases described here, although two had several bruises to the scalp indicating multiple blows to the head, two cases had only a single bruise of the frontal region and one had no bruise of the scalp at all. Each of them had bleeding in the muscles over the upper part of the side of the cervical spine, on the same side as the ruptured vertebral artery, but none had any damage to the transverse processes of the vertebrae at this level.

It seems possible that rupture of the vertebral artery in its intracranial portion may be due to the indirect application of force, as by a sudden movement of the head relative to the neck, or by a blow on the neck causing obstruction to the artery at that site, with abrupt rise in pressure in the intracranial portion. These possibilities are undergoing further study in this department.

We are grateful to Mr I Newsome for technical assistance with this study.

\section{References}

' Simonsen J. Fatal sub-arachnoid haemorrhage in relation to minor head injuries. J Forensic Med 1967; 14:146-55.

${ }^{2}$ Contostavlos DL. Massive sub-arachnoid haemorrhage due to laceration of the vertebral artery associated with a fracture of the transverse process of the atlas. J Forensic Sci $1971 ; 16: 40-56$.

${ }^{3}$ Cameron J, Mant K. Fatal sub-arachnoid haemorrhage associated with cervical trauma. Med Sci Law 1972;12:66-70.

${ }^{4}$ Gee DJ. Traumatic sub-arachnoid haemorrhage. Proceedings of the 12th congress of the International Academy of Forensic and Social Medicine, Vienna: Egermann, May 1982:495-8.

${ }^{5}$ Krauland W. Die Traumatische Subarachnoidal Blutung. $Z$ Rechtsmed 1981;87:1-18.

- Harland WA, Pitts JF, Watson AA. Traumatic sub-arachnoid haemorrhage due to upper cervical trauma. J Clin Pathol 1983;36: $1335-41$.

7 Wilkinson IMS. The vertebral artery. Extracranial and intracranial structure. Arch Neurol 1972;27:392-6.

${ }^{8}$ Piffer CR, Zorzetto NL. Microscopical anatomy of the vertebral artery in the suboccipital and intracranial segments. Anat Anz Jena 1980; 147:382-8.

- Tatsuno Y, Lindenberg R. Basal subarachnoid hematomas as sole intracranial traumatic lesions. Arch Pathol 1974;97: 211-5.

Requests for reprints to: Dr DJ Gee, Department of Forensic Medicine, Clinical Sciences Building, St James's University Hospital, Beckett Street, Leeds LS9 7TF, England. 